NASA Technical Memorandum 106347

AIAA-93-0813

\title{
Modal Element Method for Potential Flow in Non-Uniform Ducts: Combining Closed Form Analysis With CFD
}

Kenneth J. Baumeister and Joseph F. Baumeister

Lewis Research Center

Cleveland, Ohio

Prepared for the

32nd Aerospace Sciences Meeting and Exhibit

sponsored by the American Institute of Aeronautics and Astronautics

January 10-13, 1994, Reno, Nevada

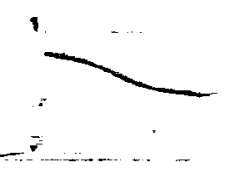

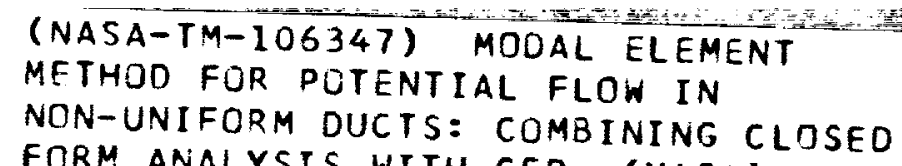

$+\infty$

N94-15799

Unclas

$63 / 64 \quad 0191159$ 


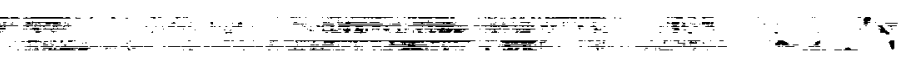




\title{
MODAL ELEMENT METHOD FOR POTENTIAL FLOW IN NONUNIFORM DUCTS:
}

\section{COMBINING CLOSED FORM ANALYSIS WITH CFD}

\author{
Kenneth J. Baumeister and Joseph F. Baumeister \\ National Aeronautics and Space Administration \\ Lewis Research Center \\ Cleveland, Ohio $\mathbf{4 4 1 3 5}$
}

\begin{abstract}
$\underline{\text { Abstract }}$
An analytical procedure is presented, called the modal element method, that combines numerical grid based algorithms with eigenfunction expansions developed by separation of variables. A modal element method is presented for solving potential flow in a channel with two-dimensional cylindrical like obstacles. The infinite computational region is divided into three subdomains; the bounded finite element domain, which is characterized by the cylindrical obstacle and the surrounding unbounded uniform channel entrance and exit domains. The velocity potential is represented approximately in the grid based domain by a finite element solution and is represented analytically by an eigenfunction expansion in the uniform semi-infinite entrance and exit domains. The calculated flow fields are in excellent agreement with exact analytical solutions. By eliminating the grid surrounding the obstacle, the modal element method reduces the numerical grid size, employs a more precise far field boundary condition, as well as giving theoretical insight to the interaction of the obstacle with the mean flow. Although the analysis focuses on a specific geometry, the formulation is general and can be applied to a variety of problems as seen by a comparison to companion theories in aeroacoustics and electromagnetics.
\end{abstract}

\section{$\underline{\text { Introduction }}$}

Computational fluid dynamics (CFD) now plays a major role in aeronautical research and design. Pironneau (1989) reports that for Dassault industries 1986 was the year when the numerical budget over took the budget for experimentation in wind tunnels. This comparison of budgets is appropriate since both wind tunnels and CFD analysis provide similar information about the flow physics. Numerical grid based solutions with color graphics and computer generated movies are closely akin to their experimental counterparts with smoke streamlines, shadowgraph, and schlieren photography. Current CFD programs in aeronautics model with incredible accuracy the flow field of whole aircraft as well as propulsion systems and rotor dynamics (Henne, 1990). Nevertheless, current CFD analysis does not develop the mathematical insight into the role of key variables and parameters that readily unfold from exact and approximate analytical solutions. Lighthill (1989) addressed this inherent deficiency when he stated that it is "essential to stress the connections between theoretical analysis on one hand and experimental and/or CFD studies on the other."

To gain more theoretical insight in CFD problems, the present paper presents an analytical procedure, called the modal element method, that combines numerical grid based algorithms with eigenfunction expansions derived by separation of variables. Herein, a modal element method is developed for solving potential flow in an infinitely long channel with a two-dimensional cylindrical like obstacle present. Emphasis is on the problem formulation. Wholly numerical finite element solutions for the streamlines and potential lines over a cylinder in a duct are presented in many introductory texts on finite elements, such as Hinton and Owen (1779, p. 222) and Segerlind (1976, p. 183), as well as advanced fluid dynamic texts such as Chung (1978, p. 177). However, the modal element method adds theoretical insight to both the numerical formulation and the physical problem. In the numerical analysis, the method will aid judgment in choosing the grid density as well as the accuracy of the exit boundary condition. In the flow problem, the method will determine the physical parameters which dictate the change of the flow streamlines and the potential lines.

Although the analysis focuses on a specific geometry, the formulation is quite general and can be applied to a variety of problems as seen by a comparison to its companion theories in acoustics and electromagnetics. In CFD applications, however, singularity requirements introduce some differences from the previous wave propagation formulations. Historically, a primary reason for developing the modal element method was to accurately describe the radiation boundary condition at the computational boundary of a numerical grid. In electromagnetics, Chang and Mei (1976), Lee and Cendes (1987), and Baumeister and Kreider (1993) applied the method to scattering from dielectric cylinders while Baumeister (1991) applied the method to electromagnetic propagation in ducts with surface irregularities. In acoustics, Astley and Eversman (1981) employed the method in duct propagation problems while Baumeister and Kreider (1992) have applied the 
technique to scattering from soft and rigid bodies. For validation, numerical calculations using the modal element method for sound propagation in a variable area duct with a cylindrical like obstacle show excellent agreement with experimental results (Baumeister, et al., 1983).

To illustrate the advantage of coupling analytic and grid based numerical solutions in the modal element method, consider the problem of finding the pressure amplitude resulting from scattering of an acoustic plane wave by a rigid cylinder. As shown in Fig. 1(a), a conventional finite difference theory (Khan, Brown, and Ahuja, 1986) requires a large dense grid to resolve the wave like nature of the pressure field and to accurately approximate the far field radiation boundary condition. In contrast, the limiting case of modal element method for rigid bodies requires only a single line of elements as shown in Fig. 1(b). Figure 1(c) compares the pressure amplitude of these analyses with the exact theoretical results shown by the solid line. The modal element method shows excellent agreement with the theory while the conventional finite difference theory shows some error because of the previously alluded approximations. The modal element method can also be extended very easily to higher frequencies, as shown in Fig. 1(d), since no nodes are required in the far field.

Considering open systems as in Fig. 1, the modal element method is a grid based numerical system that has many advantages of the classical boundary integral methods such as the boundary element method in acoustics, the panel method in aerodynamics, and the method of moments in electromagnetics. These boundary integral methods are well suited for solving the scattering problem discussed in Fig. 1. However, for the semiinfinite duct problem considered herein, the boundary element method requires a closure approximation in the far field similar to the standard finite element method (Brebbia, 1978, p. 80). Also, similar to the modal element method considered herein, the finite element method and the boundary element method can be combined as discussed by Brebbia $(1978$, p. 178) but without the modal element advantage of obtaining a closed form analytical solution. Yet in a broader sense, the modal element method could be considered a subset of the boundary element method under the title of indirect method of analysis (Brebbia, 1978, p. 2).

The motivation for adapting the modal element method for CFD analysis herein is threefold: first, to explicitly determine the importance of physical parameters by obtaining a closed form analytical solution in part of the solution domain, second, to minimize the size of the regions requiring numerical grids, and thirdly, to more accurately approximate the exit boundary condition of the numerical grid. The later consideration can be important in the direct computation of aerodynamic sound from unsteady flows where the sound levels can be three orders of magnitude smaller than mean flow variables.

In the present paper, first the method of analysis and domain decomposition is discussed followed by a development of the analytical solution. Next, subdomain interface conditions are presented followed by the finite element solution procedure and the requirements of a Dirichlet boundary condition. The geometric model and an exact solution for the model are presented next followed by results and comparisons that include two example calculations.

\section{Nomenclature}

A

$A^{(e)}$ total dimensionless area of finite element domain

$\mathrm{A}_{\mathrm{m}}^{-}$modal amplitude, Eq. (14)

$\mathrm{B}_{\mathrm{m}}^{+} \quad$ modal amplitude, Eq. (17)

b dipole strength, Eq. (37)

$c_{m}^{-} \quad$ modal amplitude, Eq. (9)

$c_{\text {m }}^{+}$modal amplitude, Eq. (9)

F column vector, right hand side of global matrix equation

h dimensionless channel height, $h^{\#} / L^{\#}$

i $\quad \sqrt{-1}$

K global matrix, Eq. (33)

$\mathrm{k}^{\#} \quad$ wave number, Fig. 1

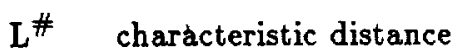

m mode number

m mode number, Eq. (19)

M number of elements in finite element domain

$M_{\text {coef number of modal coefficients used in }}$ eigenfunction expansion

$M_{p t s}$ number of grid points on interface $S$ used in integration, Eq. (20)

$\mathrm{N}$ number of nodes in finite element domain

$\mathrm{N}_{\mathrm{S}}$ number of nodes on $\mathrm{S}$ to resolve harmonics

$\mathrm{N}^{(e)}$ local linear triangular interpolation function, $\mathrm{N}^{(e)}(\mathrm{x}, \mathrm{y}) ; \mathrm{N}_{\mathrm{i}}^{(e)}\left(\mathrm{x}_{\mathrm{j}}, \mathrm{y}_{\mathrm{j}}\right)=\delta_{\mathrm{i}, \mathrm{j}}(\mathrm{i}=1,2,3 ; \mathrm{j}=1,2,3)$ outward unit normal vector

normal vector 


$\begin{array}{lll}\mathbf{R}_{\mathbf{I}} & \text { global residual error at node } \mathrm{I} & \mathrm{J} \\ \mathbf{r}^{\#} & \text { radius, Fig. 1 } & \mathrm{j} \\ \mathbf{S} & \text { line interface about finite element domain } & \mathrm{S} \\ \mathbf{S}_{\mathbf{a}} & \text { entrance plane } & \\ \mathrm{S}_{\mathrm{b}} & \text { exit plane } & \\ \mathbf{S}^{+} & \text {region exterior to } \mathrm{S} & \\ \mathrm{S}^{-} & \text {region interior to } \mathrm{S} & \\ \mathbf{S} & \text { arc length parameter on } \mathrm{S} \\ \mathrm{U}_{\infty}^{+} & \text {free stream velocity } \\ \mathrm{U}_{0}^{\#} & \text { normalizing velocity } \\ \mathrm{W}_{\mathrm{I}} & \text { global weight function associated with node } \mathrm{i} ;\end{array}$

$W_{\text {I }}$ global weight function associated with node $i$; $W_{I}\left(x_{J}, y_{J}\right)=\delta_{I J}(I=1 \ldots N ; J=1 \ldots N)$

$\mathrm{W}_{\mathrm{I}}^{(e)}$ local weight function associated with node $\mathrm{I}$

$W_{m^{*}}$ interface weight function, Eq. (19)

$\mathrm{W}(\mathrm{z})$ complex potential, Eq. (37)

$X$ separation function, Eq. (4)

$x$ dimensionless axial distance, $x^{\#} / L^{\#}$

$x_{\text {in }} \quad$ starting position of finite element grid

$x_{0} \quad$ axial intercept of obstacle

$x_{\text {out }}$ ending position of finite element grid

$\mathrm{Y}$ separation function, Eq. (4)

y dimensionless transverse distance, $y^{\#} / L^{\#}$

$y_{0}$ height of obstacle

z complex variable, Eq. (38)

B angle between element outward normal and positive $x$ axis

$\nabla^{2} \quad$ Laplace operator

$\delta_{\mathrm{IJ}} \quad$ Kronecker delta $\left(\delta_{\mathrm{IJ}}=1\right.$ for $\mathrm{I}=\mathrm{J} ; \delta_{\mathrm{IJ}}=0$ for $\mathrm{I} \neq \mathrm{J}$ )

$\lambda$ eigenvalue, Eq. (8)

$\Phi \quad$ column solution vector, Eq. (33)

$\phi \quad$ potential

$\psi \quad$ stream function

Subscripts
a analytical solution
b analytical solution
I global node index in finite element domain
$I_{s}$ global node index for interface $\mathrm{S}$
i local element mode index

J global node index, Eq. (25)

j local element node index

Superscripts

$\begin{array}{ll}\text { \# } & \text { approximate solution } \\ \overline{(1)} & \text { average value } \\ \text { (e) } & \text { element value }\end{array}$

Method of Analysis

The present study is concerned with computing the potential flow field in a channel with two-dimensional cylindrical like obstacles, as shown in Fig. 2. A uniform velocity profile $\mathrm{U}_{\infty}^{+}$is assumed to exist far upstream. For inviscid and irrotational two-dimensional flow, the potential equation governs;

$$
\nabla^{\# 2} \phi^{\#}=0
$$

where \# denotes a dimensional variable. For this paper the following dimensionless variables are introduced:

$$
\begin{gathered}
\mathbf{x}=\frac{\mathbf{x}^{\#}}{\mathrm{~L}^{\#}} \quad \mathrm{y}=\frac{\mathrm{y}^{\#}}{\mathrm{~L}^{\#}} \\
\mathbf{h}=\frac{\mathrm{h}^{\#}}{\mathrm{~L}^{\#}} \quad \mathrm{U}_{\infty}^{+}=\frac{\mathrm{U}_{\infty}^{+\#}}{\mathrm{U}_{0}^{\#}} \\
\phi=\frac{\phi^{\#}}{\mathrm{U}_{0}^{\#} \mathrm{~L}^{\#}}
\end{gathered}
$$

The superscript + indicates the direction of the velocity in the $+x$ direction. All symbols are defined in the nomenclature. Equation (1) becomes

$$
\nabla^{2} \phi=0
$$

The exact shape of the obstacle is defined by an infinite row of doublets transverse to a uniform flow (Kirchhoff, 1985). For obstacles less than half the height of the duct, its shape is nearly a circular cylinder. The advantage of using this obstacle is that an exact solution exists for validating the theoretical results. The detailed shape of the obstacle will be full described in a later section of this report.

The common numerical approach to this problem is to extend the grid far upstream and downstream of the 
obstacle such that the assumption of uniform velocity is valid, as shown in Fig. 3. Generally, a large entrance and exit grid region must be selected. The leading edge of the obstacle is defined by $x_{0}$ while the inlet and exit positions of the grid are defined by $x_{\text {in }}$ and $x_{\text {out }}$ respectively. Symmetry about the cylinder was not used allowing the computer program to handle a greater variety of problems.

In contrast to the conventional approach, a typical modal element grid system is shown in Fig. 4. The spatial domain is divided into three subdomains, the entrance and exit analytical domains and the finite element domain. The finite element domain contains a nodal grid system that covers the region of complicated geometry. Linear triangular elements are used and the subdomain interface is approximated by piecewise linear segments. In the finite element domain, an approximate solution for the velocity potential is calculated by the Galerkin method. In the analytical domains, which extends to $\pm \infty$, an eigenfunction expansion for the velocity potential is derived by separation of variables.

The modal element method couples the analytical and numerical solutions by imposing continuity on the potential and velocity at the interface between the subdomains. This coupling results in a single matrix equation in which the eigenfunction coefficients and the potential at the finite element nodes are calculated simultaneously, yielding a global representation of the potential field. Next, Eq. (3) will be solved by the method of separation of variables in the entrance and exit regions and then by the finite element technique in the grid system surrounding the obstacle.

\section{$\underline{\text { Analytical Solution }}$}

Consider the entrance portion of the duct as shown in Fig. 4 for $x<x_{\text {in }}$ that is upstream to the duct obstruction. Employing separation of variables for the solution of Eq. (3),

$$
\phi_{\mathrm{a}}=\mathrm{X}(\mathrm{x}) \mathrm{Y}(\mathrm{y})
$$

yields

$$
\frac{1}{\mathrm{X}} \frac{\partial^{2} \mathrm{X}}{\partial \mathrm{x}^{2}}=-\frac{1}{\mathrm{Y}} \frac{\partial^{2} \mathrm{Y}}{\partial \mathrm{y}^{2}}=\lambda^{2}
$$

The subscript a denotes the analytical solution in the entrance portion of the duct, as labeled in Fig. 4. Solving the ordinary differential equation for $\mathrm{Y}$ and applying the solid wall boundary conditions,

$$
\mathrm{v}=\frac{\partial \phi_{\mathrm{a}}}{\partial \mathrm{y}}=0 \quad \text { at } \quad \mathrm{y}=0 \quad \text { and } \quad \mathrm{y}=\mathrm{h}
$$

yields

$$
\phi_{\mathrm{a}}=c_{\mathrm{m}} \cos (\lambda \mathrm{y}) \mathrm{X}(\mathrm{x})
$$

where the eigenvalues are

$$
\lambda=\frac{\mathrm{m} \pi}{\mathrm{h}} \quad \mathrm{m}=0,1,2,3, \ldots
$$

Now, solving the ordinary differential equation of $\mathrm{X}$ yields (noting the double root at $m=0$ )

$$
\begin{aligned}
\phi_{a}= & {\left[c_{0}^{+} x+\sum_{m=1}^{M_{\text {coef }}} c_{m}^{+} e^{\frac{-m \pi x}{h}} \cos \left(\frac{m \pi y}{h}\right)\right] } \\
+ & {\left[c_{0}^{-}+\sum_{m=1}^{M_{\text {coef }}} c_{m}^{-e^{\frac{m \pi x}{h}}} \cos \left(\frac{m \pi y}{h}\right)\right] }
\end{aligned}
$$

where $M_{\text {coef }}$ the number of coefficients nsed in the expansion, must be set a priori. From now on, the eigenfunction terms are called modes, as commonly used in acoustic theory. The two separate roots have been distinguished by $a+$ or - superscript. For large values of negative $x$, the upstream velocity boundary condition requires

$$
\frac{\partial \phi_{\mathrm{a}}}{\partial \mathrm{x}}=\mathrm{U}_{\infty}^{+} \quad \mathrm{x} \rightarrow-\infty
$$

thus, all values of $c_{m}^{+}$must be set to zero and Eq. (9) becomes

$$
\phi_{\mathrm{a}}=\mathrm{U}_{\infty}^{+} \mathrm{x}+\left[\mathrm{c}_{\mathrm{o}}^{-}+\sum_{\mathrm{m}=1}^{\mathrm{M}_{\text {coef }}} \mathrm{c}_{\mathrm{m}}^{-} \mathrm{e}^{\frac{\mathrm{m} \pi \mathrm{x}}{\mathrm{h}}} \cos \left(\frac{\mathrm{m} \pi \mathrm{y}}{\mathrm{h}}\right)\right]
$$

Physically, the constant $\mathrm{c}_{\mathrm{o}}^{-}$in Eq. (11) represents a negative potential that will later be shown to be proportional to the size of the obstacle in the duct. The last term in Eq. (11) represents damped higher order modes that blend the distorted potential around the obstacle into the uniform potential upstream.

For convenience, the constant $c_{0}^{-}$will be pulled into the exponential, so that the summation begins with $\mathrm{m}=0$ such that 


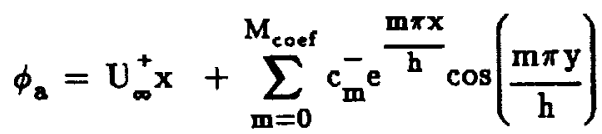

Also, because the damped exponentials can be very small at the beginning of the analytical regions, the separation constants are redefined. By introducing the identity in the brackets [],

$\phi_{\mathrm{a}}=\mathrm{U}_{\infty}^{+} \mathrm{x}+\sum_{\mathrm{m}=0}^{\mathrm{M}_{\text {coef }}} \mathrm{c}_{\mathrm{m}}^{-}\left[e^{\frac{m \pi x_{i n}}{\mathrm{~h}}} \mathrm{e}^{-\frac{m \pi x_{i n}}{\mathrm{~h}}}\right] \mathrm{e}^{\frac{\mathrm{m} \pi x}{\mathrm{~h}}} \cos \left(\frac{\mathrm{m} \pi \mathrm{y}}{\mathrm{h}}\right)$

Defining the constant $A_{m}^{-}$as

$$
A_{m}^{-}=c_{m}^{-} e^{\frac{m \pi x_{i n}}{h}}
$$

such that

$\phi_{\mathrm{a}}=\mathrm{U}_{\infty}^{+} \mathrm{x}+\sum_{\mathrm{m}=0}^{\mathrm{M}_{\text {coef }}} \mathrm{A}_{\mathrm{m}}^{-} \mathrm{e}^{\frac{\mathrm{m} \pi\left(\mathbf{x}-\mathbf{x}_{\mathrm{in}}\right)}{\mathrm{h}}} \cos \left(\frac{\mathrm{m} \pi \mathrm{y}}{\mathrm{h}}\right)$

Finally, the characteristic length $L^{\#}$ is set equal to $h^{\#}$ so that $h=1$ and Eq. (15) simplifies to

$$
\phi_{a}=U_{\infty}^{+} x+\sum_{m=0}^{M_{\text {coef }}} A_{m}^{-} e^{m \pi\left(x-x_{i n}\right)} \cos (m \pi y)
$$

Similarly, for the exit duct where $x>x_{\text {out }}$, the analytical expression for the potential becomes

$$
\phi_{\mathrm{b}}=\mathrm{U}_{\infty}^{+} \mathrm{x}+\sum_{\mathrm{m}=0}^{\mathrm{M}_{\text {coef }}} \mathrm{B}_{\mathrm{m}}^{+} \mathrm{e}^{-\mathrm{m} \pi\left(\mathrm{x}-\mathrm{x}_{\text {out }}\right)} \cos (\mathrm{m} \pi \mathrm{y})
$$

In a typical analytical solution, the separation constants $\mathrm{A}_{\mathrm{m}}^{-}$and $\mathrm{B}_{\mathrm{m}}^{+}$are evaluated by some orthogonality condition in coordinate systems that matches the physical boundary. The rectangular coordinate system chosen herein obviously does not match the circular boundary of the obstacle. However, the numerical grid system will transfer the necessary information such that $\mathrm{A}_{\mathrm{m}}^{-}$and $\mathrm{B}_{\mathrm{m}}^{+}$can be conveniently determined.

\section{Interface Conditions}

At the interface $\mathrm{S}$ between the finite element domain and the analytical domains, both the potential and velocity are continuous. The local continuity of potential

$$
\left.\phi\right|_{\mathbf{S}^{+}}-\left.\phi\right|_{\mathbf{S}^{-}}=0
$$

can be expressed numerically by a collocation procedure (Lee and Cendes, 1987) or an integral weighting procedure (Baumeister and Krieder, 1992). The latter are used here with weight functions $\cos (m \pi y)$, so the continuity of potential at the entrance interface $S_{a}$ is expressed by

$$
\begin{gathered}
\oint_{\substack{S_{a} \\
y=0}}^{y=1} W_{m^{*}}\left[\phi_{a}-\phi\right] d s=0 . \\
W_{m^{*}}=\cos \left(m^{*} \pi y\right) \\
\left(m^{*}=0,1,2, \ldots ., M_{\text {coef }} \text { equations }\right)
\end{gathered}
$$

There must be one equation for every unknown separation constant. Thus, $M_{\text {coef }}+1$ weight equations are required. The superscript ${ }^{*}$ designates the particular weight index to differentiate it from the eigenvalue index $\mathrm{m}$ used in Eq. (8). In contrast to $\mathrm{FE}$ weighted residual theory using local weight functions, the weight function here is global in nature acting over the whole y domain.

Rather than take advantage of the orthogonality conditions in Eq. (19), a completely numerical approach has been adopted to determine equations for the $A_{m}^{+}$in anticipation of future code complications. It suffices to apply a simple quadrature to obtain acceptable results when approximating Eq. (19). $S_{a}$ is divided into subintervals centered at points $\left(\mathrm{x}_{\mathrm{Is}}, \mathrm{y}_{\mathrm{Is}}\right)$, which correspond to finite element boundary nodes introduced later in the paper. The nodes are evenly spaced on the boundary with the index $I_{s}$. Once the number of modes $M_{\text {coef }}$ has been assumed (based on convergence of the numerical solution) the grid is set up so that the number of nodes on $\mathrm{S}_{\mathrm{a}}$ is $\mathrm{N}_{\mathrm{S}} \geq 6 \mathrm{M}_{\text {coef }}$ to adequately resolve all the harmonic terms in Eq. (16).

Applying the trapezoidal rule to the chosen nodes gives 


$$
\begin{gathered}
\sum_{I_{2}=1}^{M_{p t s}}\left[\phi_{a}\left(x_{i n}, y_{I_{a}}\right)-\phi_{I}\right] \cos \left(m^{*} \pi y_{I_{a}}\right) \Delta y_{I_{a}}=0 \\
\left(m^{*}=0,1,2, \ldots ., M_{\text {coef }} \text { equations }\right)
\end{gathered}
$$

where $\Delta y$ is the distance between nodes except at the two end points. At the ends, $\Delta y$ is half the distance between nodes. Thus,

$$
\begin{aligned}
\Delta \mathrm{y}_{\mathrm{I}_{s}} & =\frac{1-\frac{1}{2}\left(\delta_{\mathrm{I}_{\mathrm{s}} 1}+\delta_{\mathrm{I}_{\mathrm{q}} \mathrm{M}_{\mathrm{pts}}}\right)}{\mathrm{M}_{\mathrm{pts}}-1} \\
\delta & =\text { Kronecker } \Delta
\end{aligned}
$$

By expressing $\phi_{a}$ in terms of the modal coefficients in Eq. (16), Eq. (20) can be written explicitly as

$$
\begin{aligned}
& \sum_{m=0}^{M_{\text {coef }}} A_{m}^{-} \sum_{I_{s}=1}^{M_{p t !}} \cos \left(m \pi y_{I_{g}}\right) \cos \left(m^{*} \pi y_{I_{1}}\right) \Delta y_{I_{1}} \\
& -\sum_{I_{s}=1}^{M_{p t s}} \cos \left(m^{*} \pi y_{I_{z}}\right) \phi_{I_{z}} \Delta y_{I_{s}} \\
& =-U_{\infty}^{+} x_{i} \sum_{I_{0}=1}^{M_{p t s}} \cos \left(m^{*} \pi y_{I_{s}}\right) \Delta y_{I_{s}} \\
& \left(\mathrm{~m}^{*}=0,1,2, \ldots, \mathrm{M}_{\text {coef }}\right. \text { equations) }
\end{aligned}
$$

Equation (22) comprises $M_{\text {coef }}+1$ separate difference equations, each of which is written in terms of all the unknown coefficients $A_{m}^{-}$and the potential $\phi_{l s}$ at the nodes on $\mathrm{S}_{\mathbf{a}}$.

The continuity of velocity requires that at the interface

$$
\left.\frac{\partial \phi_{\mathrm{a}}}{\partial \mathrm{n}}\right|_{\mathrm{s}^{+}}=\left.\frac{\partial \phi}{\partial \mathrm{n}}\right|_{\mathrm{s}^{-}}
$$

where $\mathbf{n}$ is the outward normal. This relationship is used to satisfy the natural boundary condition later required in the finite element portion of the problem.

A similar set of equations can be written for the outlet region coefficients $B_{m}^{+}$. These equations are later combined with the finite element equations to form a matrix system that yields values of all the unknowns $\phi_{I}$ values at the nodes as well as the separation constants $A_{m}^{-}$and $B_{m}^{+}$.

\section{Finite Element Solution}

The finite element domain, with total area $A$, is divided into $M$ discrete triangular elements, $A^{(e)}$, $e=1,2, \ldots, M$, defined by $N$ corner nodal points $\left(x_{1}, y_{I}\right)$, $I=1,2, \ldots, N$. The corner nodes for element area $A(e)$ are denoted $\left(x_{1}^{(e)}, y_{1}^{(e)}\right),\left(x_{2}^{(e)}, y_{2}^{(e)}\right)$, and $\left(x_{3}^{(e)}, y_{3}^{(e)}\right)$.

The potential is approximated by a linear combination of weight functions $W_{1}(x, y)$ :

$$
\tilde{\phi}(\mathrm{x}, \mathrm{y})=\sum_{\mathrm{I}=1}^{\mathrm{N}} \mathrm{W}_{\mathrm{I}}(\mathrm{x}, \mathrm{y}) \phi_{\mathrm{I}}=[\mathrm{W}(\mathrm{x}, \mathrm{y})]\{\phi\},
$$

with [] representing a row vector and \{\} representing a column vector. The weights have the property that

$$
\mathrm{W}_{\mathrm{I}}\left(\mathrm{x}_{\mathrm{J}}, \mathrm{y}_{\mathrm{J}}\right)=\delta_{\mathrm{IJ}} \quad(\text { Kronecker } \delta)
$$

so that the unknown nodal pressure values are given by $\phi_{\mathrm{I}}=\tilde{\phi}\left(\mathrm{x}_{\mathrm{I}}, \mathrm{y}_{\mathrm{I}}\right)$.

To determine $\{\phi\}$, apply the method of weighted residuals. In this method, the residual error of Eq. (3),

$$
\begin{aligned}
& \mathrm{R}_{\mathrm{I}}=\iint_{\mathrm{A}} \mathrm{W}_{\mathrm{I}}(\nabla \cdot \nabla \tilde{\phi}) \mathrm{d} \mathbf{x} \mathrm{dy} \\
& (\mathrm{I}=1,2,3, \ldots, \mathrm{N} \text { one residual } \\
& \text { equation for each node } \mathrm{I})
\end{aligned}
$$

is set to 0 for each node I. Applying Green's vector identity (integration by parts) and the divergence theorem to the integrand in Eq. (26) yields the weak formulation of Eq. (3):

$$
\begin{gathered}
\mathrm{R}_{\mathrm{I}}=\iint_{\mathrm{A}}\left(\nabla \mathrm{W}_{\mathrm{I}} \cdot \nabla \tilde{\phi}\right) \mathrm{d} \mathbf{x} \mathrm{dy}-\int_{\mathrm{S}}\left(\mathrm{W}_{\mathrm{I}} \nabla \tilde{\phi} \cdot \overline{\mathrm{n}}\right) \mathrm{ds}=0 . \\
(\mathrm{I}=1,2,3, \ldots, \mathrm{N})
\end{gathered}
$$

Equation (27) is a global, or node-oriented, formulation, in that it provides a difference equation for each node that can be used to determine $\{\phi\}$.

From a practical standpoint, though, it is more convenient to consider a local, or element-oriented, formulation. To develop the local formulation, write each residual $R_{I}$ as the sum of the element residuals: 


$$
\begin{aligned}
& 0=R_{I}=\sum_{e=1}^{M} R_{I}^{(e)}= \sum_{e=1}^{M}\left[\iint_{A^{(e)}}\left(\nabla W_{I}^{(e)} \cdot \nabla \tilde{\phi}^{(e)}\right) d A\right. \\
&\left.-\int_{S^{(e)} n s}\left(W_{I}^{(e)} \nabla \tilde{\phi}^{(e)} \cdot \bar{n}\right) d s\right] \\
&(I=1,2,3, \ldots, N)
\end{aligned}
$$

where $S^{(e)}$ is the boundary of element $A^{(e)}$.

In the boundary integral terms in Eq. (28), it is reasonable to approximate the (continuous) normal derivative with its mean value over $S^{(e)} \cap S$. The key step is to apply the continuity of velocity (Eq. (23)), which introduces the eigenfunction coefficients, thus linking the analytic solution and the finite element solution on the interface. The term is transformed as follows:

$$
\begin{aligned}
\int_{S^{(e)} \cap S}\left\langle W_{I}^{(e)} \nabla \tilde{\phi}^{(e)} \cdot \bar{n}\right) d s & =\int_{S^{(e)} \cap S}\left(W_{I}^{(e)} \frac{\partial \tilde{\phi}^{(e)}}{\partial n}\right) d s \\
& =\left(\frac{\partial \phi_{a}}{\partial n}\right)_{S^{(e)}} \int_{S^{(e)} \cap s_{a}} W_{I}^{(e)} d s \\
& +\left(\overline{\frac{\partial \phi_{b}}{\partial n}}\right)_{S^{(e)}} \int_{S^{(e)} \cap S_{b}} W_{I}^{(e)} d s,
\end{aligned}
$$

Only the entrance and exit interfaces contribute, since the normal derivative of the potential is zero along the upper and lower channel walls and the obstacle.

If $B$ is the angle between the positive $x$ axis and the outward normal, for the geometry shown in Fig. 4, the interface between the elements and the analytical regions is vertical and $B=180^{\circ}$ at the entrance; thus, the normal derivative can be simplified to

$$
\left(\overline{\frac{\partial \phi_{\mathrm{a}}}{\partial \mathrm{n}}}\right)_{\mathrm{S}(\mathrm{e})}=\left(\overline{\frac{\partial \phi_{\mathrm{a}}}{\partial \mathrm{x}} \cos (\beta)+\frac{\partial \phi_{\mathrm{a}}}{\partial \mathrm{y}} \sin (\beta)}\right)_{\mathrm{S}(\mathrm{e})}=\left(\overline{-\frac{\partial \phi_{\mathrm{a}}}{\partial \mathrm{x}}}\right)_{\mathrm{S}^{(e)}}
$$

Similar for the exit except $B=0$ so that the $\cos (B)$ has a value of +1 .

Substituting Eqs. (29) and (30) into Eq. (28) yields

$$
\begin{aligned}
& 0=R_{I}=\sum_{e=1}^{M} {\left[\iint_{A^{(e)}}\left(\nabla W_{I}^{(e)} \cdot \nabla \tilde{\phi}^{(e)}\right) d A\right.} \\
&-\left(-\frac{\partial \phi_{a}}{\partial x}\right)_{S^{(e)}} \int_{S^{(e)} \cap s_{s}} W_{I}^{(e)} d s \\
&\left.-\left(\overline{\frac{\partial \phi_{b}}{\partial x}}\right)_{S^{(e)}} \int_{S^{(e)} \cap S_{b}} W_{I}^{(e)} d s\right] . \\
&(I=1,2,3, \ldots, N)
\end{aligned}
$$

The gradient of the potential in Eq. (31) can be evaluated directly from Eqs. (16) and (17) in terms of the unknown amplitude coefficients $\mathrm{A}^{-}$and $\mathrm{B}^{+}$.

To evaluate the integrals in Eq. (31), it is necessary to represent $\tilde{\phi}(x, y)$ locally. Let $N_{j}(e), j=1,2,3$, be the local linear shape functions for linear triangular elements associated with each corner node (Segerlind, 1976, p. 29), so that

$$
\begin{aligned}
\tilde{\phi}^{(e)}(x, y) & =N_{1}^{(e)}(x, y) \phi_{1}^{(e)}+N_{2}^{(e)}(x, y) \phi_{2}^{(e)} \\
& +N_{3}^{(e)}(x, y) \phi_{3}^{(e)} \\
& =\sum_{j=1}^{3} N_{j}^{(e)}(x, y) \phi_{j}^{(e)}=\left[N^{(e)}(x, y)\right]\left\{\phi^{(e)}\right\}
\end{aligned}
$$

Next, implement the Galerkin method. When the global index I equals the local node index $i$ associated with node $\left(x_{1}^{(e)}, y_{1}^{(e)}\right)$, let $W_{l}^{(e)}$ equal the local shape function $\mathrm{N}_{1}^{(e)}$. The global shape function $\mathrm{W}_{\mathrm{I}}$ is assumed to be identically zero for any element where node I does not appear (simple pyramid weight approximation); thus, the line integral in Eq. (28) vanishes unless node $I$ is on the boundary $\mathrm{S}$.

The solution to Eq. (31) is obtained by performing the usual element by element formation of the global matrix as presented in introductory FE texts. The final form of the global matrix equation is obtained by combining the solution of Eq. (31) along with Eq. (22) and the exit equivalent to Eq. (22).

$$
[\mathrm{K}]\{\Phi\}=\{\mathrm{F}\}
$$


where

$$
\begin{aligned}
& \{\Phi\}^{T}=\left[A_{0}^{-}, A_{1}^{-}, A_{2}^{-}, \ldots, A_{M_{\text {coef }}^{-}}^{-}\right. \\
& \left.\phi_{1}, \phi_{2}, \ldots, \phi_{N}, \mathrm{~B}_{0}^{+}, \mathrm{B}_{1}^{+}, \mathrm{B}_{2}^{+}, \ldots, \mathrm{B}_{\mathrm{M}_{\text {coef }}^{+}}^{+}\right] \text {. }
\end{aligned}
$$

F contains the free stream velocity terms present in Eq. (22) and from the free stream velocity terms that enter Eq. (31) through the derivatives of $\phi_{a}$ and $\phi_{a}$.

The matrix $\mathrm{K}$ has the following general form

$$
\mid \begin{array}{c:l:l}
A^{(11)} & \Phi^{(12)} & 0 \\
\hdashline A^{(21)} & \Phi^{(22)} & B^{(23)} \\
\hdashline 0 & \Phi^{(32)} & B^{(33)}
\end{array}
$$

The top and bottom rows in the matrix represent the contribution from Eq. (22) for the entrance and its equivalent exit representation. The middle row represents the contribution from Eq. (31).

The submatrix $A^{(11)}$ is a full $M_{\text {coef }} \times M_{\text {coef }}$ matrix composed of the coefficients of the $A_{m}^{-}$terms in Eq. (22) which resulted in applying Eq. (18) at the entrance interface. The submatrix $\Phi^{(12)}$ is a sparse $M_{\text {coef }} \times N$ matrix composed of the coefficients of $\phi_{1}$ in Eq. (22). The submatrices $\Phi^{(32)}$ and $B^{(33)}$ are similar in form resulting from applying Eq. (18) at the exit interface.

The submatrix $A^{(21)}$ is an $N \times M_{\text {coef }}$ matrix composed of the coefficients of $A_{m}^{-}$from the surface integral in Eq. (31). For each boundary node, there is a full row of terms in $A^{(21)}$. For interior nodes not on the boundary, there is a full row of zeros in $A^{(21)}$ since the surface integral in Eq. (31) does not contribute to the difference equation at the interior nodes. A similar interpretation applies to $B^{(23)} . \Phi^{(22)}$ is a sparse, highly banded $N \times N$ matrix composed of the coefficients of $\phi_{1}$ resulting from the solution of Eq. (31). Equation (33) is solved by a standard frontal solver program.

\section{Dirichlet Condition}

One additional constraint is required to keep the matrix (Eq. (35)) nonsingular; namely the potential must be given a value (grounded) at some nodal location in the finite element grid. For the purposes of this paper,

$$
\phi_{1}=0.0 \quad \text { at } \quad x=0.0 \quad y=1.0
$$

This condition is necessary because complete information about the magnitude of the analytical duct modes was not passed to the nodal difference equations. Information about the entrance condition is passed to the finite element equations through the $x$ derivative of the analytical solution in Eq. (31). However, the magnitudes of the lowest order reflected mode $A_{0}^{-}$and lowest order transmitted mode $\mathrm{B}_{\circ}^{+}$are not passed into the modal difference equations because the normal derivatives of the lowest order modes are identical to zero.

In retrospect, Eq. (36) was not required in acoustics and electromagnetic applications of the modal element method discussed in the introduction. In these applications, the Helmholtz equation governs rather than the Laplace equation. Consequently, the reflected and transmitted lowest order modes are harmonic wave like functions of $x$ and derivatives exist for all modes. Thus, sufficient information is transmitted to the finite element equations to make them nonsingular.

\section{Geometrical Model and Exact Solutions}

The cylindrical like obstacle shown in Fig. 2 is described by an infinite row of doublets in a uniform stream. Here, the complex potential can be written as (Kirchhoff, 1985)

$$
\mathrm{W}(\mathrm{z})=\phi(\mathrm{x}, \mathrm{y})+\mathrm{i} \psi(\mathrm{x}, \mathrm{y})=\mathrm{U}_{\omega^{+}} \mathrm{z}+\frac{\pi \mathrm{b}^{2} \mathrm{U}_{\infty}^{+}}{2} \operatorname{coth}\left(\frac{\pi}{2} \mathrm{z}\right)
$$

where

$$
z=x+i y
$$

and where the notation has been modified to reflect the nondimensionalization used herein. Using the identity given in Abramowitz and Stegun (1964, p. 84), the potential and stream function can be written as

$$
\begin{aligned}
& \phi=\mathrm{U}_{\infty}^{+} \mathrm{x}+\frac{\pi \mathrm{b}^{2} \mathrm{U}_{\infty}^{+}}{2} \frac{\sinh (\pi \mathrm{x})}{\cosh (\pi \mathrm{x})-\cos (\pi \mathrm{y})} \\
& \psi=\mathrm{U}_{\infty}^{+} \mathrm{y}-\frac{\pi \mathrm{b}^{2} \mathrm{U}_{\infty}^{+}}{2} \frac{\sin (\pi \mathrm{y})}{\cosh (\pi \mathrm{x})-\cos (\pi \mathrm{y})}
\end{aligned}
$$

In the examples to follow,

$$
\mathrm{U}_{\infty}^{+}=1.0
$$


so that the value of the streamline along the upper wall $y=1$ is $\psi=1$ and the value of the streamline is $\psi=0$ along the lower wall and obstacle. Therefore, the height of the obstacle $y$ as a function of $x$ is given by

$$
0=y-\frac{\pi b^{2}}{2} \frac{\sin (\pi y)}{\cosh (\pi x)-\cos (\pi y)}
$$

Equation (42) is solved numerically by the bisection method for the height $y$ of the obstacle as a function $x$ and as a function of the assumed strength of the dipole parameter $b$. The following two values of $b$ are used in the examples of the next section:

$\begin{array}{ccc}b & x_{0} & y_{0} \\ 0.5642 & 0.5084 & 0.5000 \\ 1.9020 & 1.1552 & 0.9000\end{array}$

Recall that the extremes of the obstacle $x_{0}$ and $y_{0}$ are defined in Fig. 2. For $b$ equals 0.5642 the obstacle closely approximates a circular cylinder.

Equation (39) represents the exact analytical solution to which the approximate numerical analysis will be compared graphically in the examples to follow. In addition, the numerically determined modal coefficients in Eq. (16) can also be compared to the exact results in Eq. (39) by equating both expressions:

$$
\begin{array}{r}
A_{0}^{-}+\sum_{m=1}^{M_{\text {coef }}} A_{m}^{-} e^{m \pi\left(x-x_{i n}\right)} \cos (m \pi y) \\
=\frac{\pi b^{2}}{2} \frac{\sinh (\pi x)}{\cosh (\pi x)-\cos (\pi y)}
\end{array}
$$

The lowest order reflected mode $A_{0}^{-}$can be easily determined by letting $x$ go to negative infinity. Noting that the higher order modes on the left-hand side go to zero and the ratio of $\sinh$ to $\cosh$ is -1 at $-\infty$, it follows

$$
\mathrm{A}_{0}^{-}=-\frac{\pi \mathrm{b}^{2}}{2}
$$

Thus, the lowest order potential acts with the opposite polarity of the free stream potential and is proportional to the square of the dipole strength.

The amplitude of the higher order modes can also be determined using the series expansion (Gradshteyn and Ryzhik, 1965, p. 42, Eq. (1.461), modified for $x<0$ )

$$
\begin{aligned}
& \frac{\sinh (\pi x)}{\cosh (\pi x)-\cos (\pi y)} \\
&=-1-2 \sum_{m=1}^{\infty} e^{m \pi x} \cos (m \pi y) \\
& x<0
\end{aligned}
$$

Substituting Eq. (44) into Eq. (43), the exact expression for the modal amplitude $A_{m}^{-}$can be expressed as

$$
A_{m}^{-}=-\pi b^{2} e^{m \pi x_{i n}}
$$

Equations (43) and (45) can be used to compare the exact results with the analytical approximations. A similar analysis applies to the $\mathrm{B}_{\mathrm{m}}^{+}$modes. For this symmetrical obstacle the $\mathrm{B}_{\mathrm{m}}^{+}$modes are identical in magnitude to the $A_{m}^{-}$modes but of opposite sign.

For the examples to be considered later, the following tabulated values give the exact amplitude of the back reflected potential $A_{0}^{-}$and the higher order cutoff potential modes.
Example 1

$b=0.5642$
$x_{0}=-0.5084$
$x_{\text {in }}=-0.5084$

$\mathrm{A}_{0}^{-}=-0.5000 \mathrm{E}+00$

$\mathrm{A}_{2}^{\frac{1}{2}}=-0.4099 \mathrm{E}-01$

$\mathrm{A}_{\underline{3}}^{\frac{2}{3}}=-0.8299 \mathrm{E}-02$

$\mathrm{A}_{4}^{\frac{3}{4}}=-0.1680 \mathrm{E}-02$

$\mathrm{A}_{5}^{\frac{4}{5}}=-0.3401 \mathrm{E}-03$

$\mathrm{A}_{6}^{\frac{5}{6}}=-0.6887 \mathrm{E}-04$
$\mathrm{A}_{\underline{\underline{1}}}^{\underline{\underline{0}}}=-0.2024 \mathrm{E}+00$

\section{Example 2}

$\mathrm{b}=1.9020$
$\mathrm{x}_{\mathrm{o}}=-1.1552$
$\mathrm{x}_{\mathrm{in}}=-1.3984$

$\mathrm{A}_{1}^{\underline{0}}=-0.1404 \mathrm{E}+00$

$\mathrm{A}_{\underline{2}}^{\frac{1}{2}}=-0.1736 \mathrm{E}-02$

$\mathrm{A}_{3}^{\frac{2}{3}}=-0.2146 \mathrm{E}-04$

$\mathrm{A}_{5}^{\underline{4}}=-0.3279 \mathrm{E}-08$

$\mathrm{A}_{6}^{\frac{5}{6}}=-0.4053 \mathrm{E}-10$
$\mathrm{A}_{0}^{-}=-5.6825 \mathrm{E}+00$

$\mathrm{A}_{4}^{\frac{3}{4}}=-0.2652 \mathrm{E}-06$

As seen above, the amplitudes of the higher order modes fall off rapidly with increasing $m$. Thus, the grid density can be reasonably sparse in the transverse $y$ direction to resolve the important modes. Because the grid was extended farther from the body in the second example $\left(x_{i n}<x_{0}\right)$, the modal element coefficients for the higher order modes are smaller for this second example. Of course, as the magnitude of $x_{\text {in }}$ increases to very large values, all the higher order modes will become negligible, so the grid density could be very sparse near the end of the finite element grid in the neighborhood $x_{i n}$. However, the analysis also indicates that the finite element grid density must still be increased at $x_{0}$ to whatever level of accuracy is desired to resolve the higher order modes. 


\section{Results and Comparisons}

To validate the method, two numerical experiments are presented for potential flow over a cylinder like obstacle described by Eq. (42). The first example considers an obstacle that blocks 50 percent of the channel while the second example is concerned with an obstacle blocking 90 percent of the channel. For the examples to follow, recall that the characteristic length $L^{\#}$ is set equal to the height of the channel so that the channel has a dimensionless height of unity. The dimensionless mean flow velocity is also assumed to be unity. The parameter $\mathrm{M}_{\text {coef }}$ specifying the number of modes in Eq. (8) was taken to be 3 for a good comparison to the exact solution. For problems without an exact solution, the number of modes must be increased till the results converge. For example, in the acoustic application shown in Fig. 1(d), the number of modes required was 121.

Example 1.-Half Channel Obstruction

Consider the potential flow over the cylinder with a $b$ value of $0.5642, x_{0}=-0.5082$, and $y_{0}=0.5000$. In this example, the finite element region was placed directly over the obstacle as shown in Fig. 4 so that the end of the analytical domain $x_{\text {in }}$ coincides with the beginning of the obstacle at $x_{0}$. As shown in Fig. 4, the finite element domain extends from -0.5082 to 0.5082 . Thirteen nodes were used on the interface and eleven nodes along the surface of the obstacle for a total of 143 nodes and 240 elements.

In Fig. 5, the velocity potential is plotted as a function of $x$ along the upper wall, $y=1$. The dashed line represents the potential on the duct wall without an obstacle. The modal element solutions (hollow boxes) are compared to the exact solution (solid line) given by Eq. (39). In Fig. 5, for $-0.5082<x<0.5082$, the values of potential at the finite element nodes are used to generate the solution. Here, eleven closely packed nodal values are shown in Fig. 5.

Also in Fig. 5, the numerical solution is generated from $\mathrm{Eq}$. (16) using the numerically determined modal coefficients $A_{m}^{-}$for $x<-0.5082$ and $B_{m}^{+}$for $x>0.5082$. The numerically calculated modal coefficients and the exact coefficients are:

$$
\begin{array}{lc}
\text { Numerical Solution } & \text { Exact Solution } \\
& \\
\mathrm{A}_{\underline{0}}^{-}=-0.486 \mathrm{E}-00 & \mathrm{~A}_{\overline{0}}^{-}=-0.50002 \mathrm{E}-00 \\
\mathrm{~A}_{1}^{-}=-0.197 \mathrm{E}-00 & \mathrm{~A}_{\overline{1}}^{-}=-0.20247 \mathrm{E}-00 \\
\mathrm{~A}_{2}^{-}=-0.454 \mathrm{E}-01 & \mathrm{~A}_{2}^{-}=-0.40994 \mathrm{E}-01
\end{array}
$$

The numerically calculated and exact modal coefficients are in reasonable agreement. Six separate values of the

potential were calculated in each analytical region as shown in Fig. 5. Clearly, the modal element method gives good agreement with the numerical results.

A convergence check was made in this example by increasing the number of vertical nodes to 25 and $x$ coordinate nodes to 21 for a total of 525 nodes and 960 elements. In this case, the numerically calculated modes and the exact modes are

$$
\begin{aligned}
& \mathrm{A}_{0}^{-}=-0.497 \mathrm{E}-00 \\
& \mathrm{~A}_{\frac{1}{1}}=-0.202 \mathrm{E}-00 \\
& \mathrm{~A}_{2}=-0.446 \mathrm{E}-01
\end{aligned}
$$

Numerical Solution

\section{Exact Solution}

$$
\begin{aligned}
& \mathrm{A}_{\overline{0}}^{-}=-0.50002 \mathrm{E}-00 \\
& \mathrm{~A}_{\overline{1}}=-0.20247 \mathrm{E}-00 \\
& \mathrm{~A}_{2}=-0.40994 \mathrm{E}-01
\end{aligned}
$$

No improvement of the graphical results was seen by eye. Figure 6 shows the resulting contour plot including the analytical and finite element regions. The dash line in Fig. 6 shows the streamlines. There is good agreement between the exact and the modal element results.

The disagreement in the $A_{2}^{-}$modal amplitude was believed to be a result of the highly skewed triangles near the leading edge of the cylinder as shown in Fig. 3. This simple grid system was chosen just to confine the grid directly over the obstacle. In the next example with 90 percent channel blockage and a very steep slope near the leading edge, a more conventional grid system is employed. As will now be shown, the new grid system will lead to good resolution of the highest order mode.

\section{Example 2.-Large Channel Obstruction}

Consider the potential flow over the cylinder with a $b$ value of $1.9020, x_{0}=1.1552$, and $y_{o}=0.9000$. In this case, 90 percent of the channel has an obstruction as illustrated in Fig. 7. The grid was extended slightly in front of the obstacle to better resolve the steep slope of the obstacle near $x_{0}$. In this case, $x_{i n}=-1.3984$ and $x_{\text {out }}=1.3984$. Twenty one nodes were used on the interface and 132 nodes along the upper channel wall for a total of 1272 nodes and 2212 elements, as shown in Fig. 7.

In Fig. 8, the velocity potential is again plotted as a function of $x$ along the upper wall, $y=1$. The dashed line again represents the potential on the duct wall without an obstacle. The modal element solutions (hollow boxes) are compared to the exact solution (solid line) given by Eq. (39). In Fig. 8, for $-1.3984<x<1.3984$, the values of potential at the finite element nodes are used to generate the solution. In this case, the closely packed nodal values are shown in Fig. 8.

Also in Fig. 8, the numerical solution is generated from Eq. (16) using the numerically determined modal 
coefficients $A_{m}^{-}$for $x<-1.3984$ and $B_{m}^{+}$for $x>1.3984$. The numerically calculated modal coefficients and the exact coefficients are as follows:

Numerical Solution Exact Solution

$$
\begin{array}{ll}
\mathrm{A}_{\underline{0}}^{-}=-0.567 \mathrm{E}+01 & \mathrm{~A}_{\underline{0}}^{-}=-0.56825 \mathrm{E}+01 \\
\mathrm{~A}_{\overline{1}}^{\underline{1}}=-0.140 \mathrm{E}-00 & \mathrm{~A}_{\overline{1}}=-0.14048 \mathrm{E}-00 \\
\mathrm{~A}_{2}=-0.173 \mathrm{E}-02 & \mathrm{~A}_{2}=-0.17364 \mathrm{E}-02
\end{array}
$$

In this example, the numerically calculated and exact modal coefficients are in good agreement for all modes including the highest order mode. Six separate values of the potential were calculated in each analytical region as shown in Fig. 8. Clearly, the modal element method again gives good agreement with the numerical results.

Finally, Fig. 9 shows a contour plot of the potential inside the finite element region while Fig. 10 shows a contour plot including both the analytical and finite element regions. The dash line in Fig. 10 shows the streamlines. Again, there is good agreement between the exact and the modal element results.

The first mode $\mathrm{A}_{\circ}^{-}$has increased from -0.5 to $\mathbf{- 5 . 6 8 2 5}$ or a factor of 10 increase in the magnitude of the potential. This is a direct result of the larger obstruction in the second example. In both cases the gradient of the potential along the upper wall reaches the free stream value once outside the obstacle because of the quick decay of the higher order modes.

\section{Concluding Remarks}

The modal element method for potential flow over a two-dimensional cylindrical like obstacle is presented. The total flow domain is broken into three subdomains that are patched together. The potential field is represented by a finite element solution in the irregular subdomain next to the obstacle and by an exact eigenfunction expansion in the unbounded entrance and exit ducts. The analytical and numerical solutions are coupled by the continuity of potential and velocity across the interface between the subdomains and are calculated simultaneously from a single matrix equation. The method is applicable to problems involving a complete range of channel blockage.

The combined numerical and analytical results show excellent agreement with the corresponding exact solutions. For numerical insight, the analytical results indicate the accuracy of the chosen exit boundary condition and the grid density required for a given harmonic accuracy near the obstacle (generally about 12 nodes per wavelength are required to resolve a modal harmonic). For flow field insight, the analytical results indicate the exact magnitude of the back potential and the decay rates of the harmonics which blend the flow streamline and the potential lines from about the obstacle into the uniform flow lines of the far field.

Eigenfunction solutions are applicable for a wide range of practical CFD problems in regions where viscosity no longer dominates. Nevertheless, eigenfunction solutions do not exist for most CFD problems. For these more complicated problems, a challenging and intriguing aspect of the modal element approach could be to use a finite series of known trial functions with unknown coefficients $A_{i}$. These trial functions would approximate the physics, but not necessarily satisfy the governing differential equations. Meirovitch (1967) suggests using admissible trial functions (satisfying geometric boundary conditions) or comparison trial functions (satisfying geometric and natural boundary conditions) for this task. In these cases, additional constraints must be applied to the coefficients $A_{i}$ so that differential equations are satisfied in the analytical region. Meirovitch suggests that the collocation method is a practical approach to this problem.

The long term goal or vision of this research is to (1) adapt the modal element method for greater analytical and numerical insight to a wider class of CFD problems and (2) decrease computational costs by reducing the numerical grid.

\section{References}

Abramowitz, M. and Stegun, I.A., "Handbook of Mathematical Functions With Formulas, Graphs, and Mathematical Tables," U.S. Department of Commerce, National Bureau of Standards Applied Mathematics Series, 55, June 1964.

Astley, R.J. and Eversman, W., 1981, "Acoustic Transmission in Non-Uniform Ducts with Mean Flow, Part II: The Finite Element Method," Journal of Sound and Vibration, Vol. 74, pp. 103-121.

Baumeister, K.J., Eversman, W., Astley, R.J., and White, J.W., 1983, "Acoustics in Variable Area Duct: Finite Element and Finite Difference Comparison to Experiment," AIAA Journal, Vol. 21, No 2, pp 193-199.

Baumeister, K.J., 1991, "Effect of Surface Deposits on Electromagnetic propagation in Uniform Ducts," IEEE Trans. on Magnetics, Vol. 27, No. 5, Sept. 1991.

Baumeister, K.J. and Kreider, K.L., "Modal Element Method for Scattering of Sound by Absorbing Bodies," Transactions of the ASME, Jour. Vibrations and Acoustics, Vol. 115, p 314-323, July 1993. 
Baumeister, K.J. and Kreider, K.L., "Modal Ring Method for the Scattering of Electromagnetic Waves," NASA TM-105966, Nov. 1992.

Brebbia, C.A., 1978, "The Boundary Element Method for Engineers," Pentech Press, London.

Chang, S.K. and Mei, K.K., 1976, “Application of the Unimoment Method to Electromagnetic Scattering of Dielectric Cylinders," IEEE Trans. on AP, pp. 35-42.

Chung, T.J., 1978, "Finite Element Analysis in Fluid Mechanics," McGraw-Hill, New York.

Gradshteyn, I.S. and Ryzhik, I.M., "Table of Integrals, Series, and Products," Academic Press, New York, 1965.

Henne, P.A., 1990, Applied Computational Aerodynamics, AIAA, Washington DC.

Hinton, E. and Owen, D.R.J., 1979, "An Introduction to Finite Element Computations," Pineridge Press, Swansea, U.K.

Lee, J.-F. and Cendes, Z.J., 1987, "The Transfinite Element Method for Computing Electromagnetic Scat- tering From Arbitrary Lossy Cylinders," AP-S International Symposium, paper AP03-5, Blacksburg, VA.

Lighthill, J., Panel Summary: Theory, Instability and Transition, Vol. V, M.Y. Hussaini, and R.G. Voigt, eds., Springer-Verlag, 1989, New York.

Khan, M.M.S., Brown, W.H., and Ahuja, K.K.,1986, "Computational Aeroacoustics as Applied to the Diffraction of Sound by Cylindrical Bodies," AIAA paper 86-1879.

Kirchhoff, R.H., "Potential Flows-Computer Graphic Solutions," Marcel Dekker, Inc, New York, 1985.

Meirovitch, L., "Analytical Methods in Vibrations," Macmillan Company, London, 1967.

Pironneau, O., Finite Element Methods for Fluids, John Wiley \& Sons, 1989.

Segerlind, L.J., 1976, "Applied Finite Element Analysis," John Wiley \& Sons, New York. 


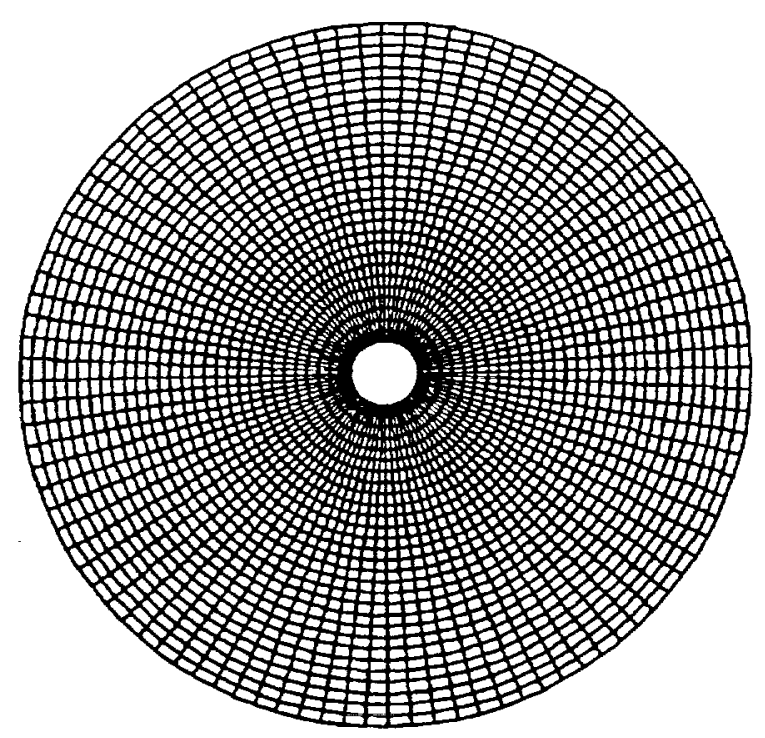

(a) Computational grid; Khan, Brown, Ahuja transient difference.
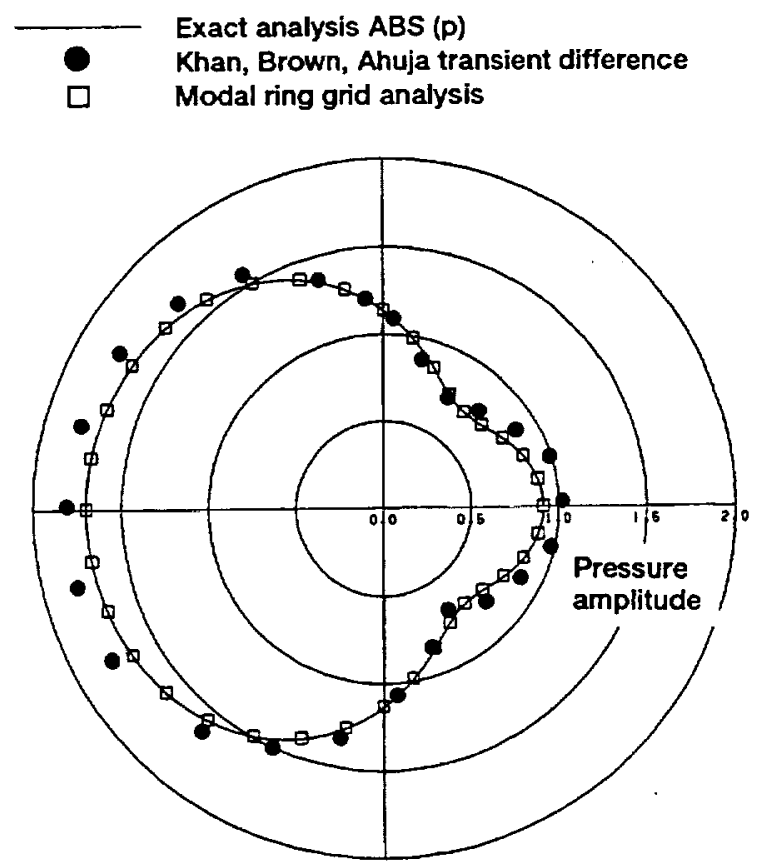

(c) Comparison of Numerical Approaches $\mathrm{k}^{\#}=$

$0.182 \mathrm{~cm}^{-1}$ radius of cylinders $=5 \mathrm{~cm}, \mathrm{k}^{\#} \mathrm{r}^{\#}=1.08$.

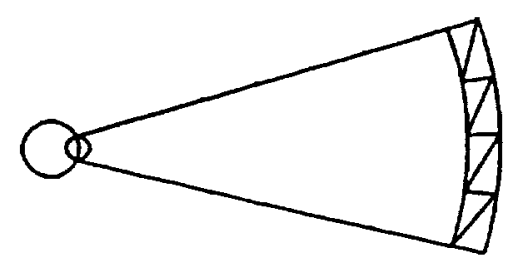

radius of cylinder $=5 \mathrm{~cm}$
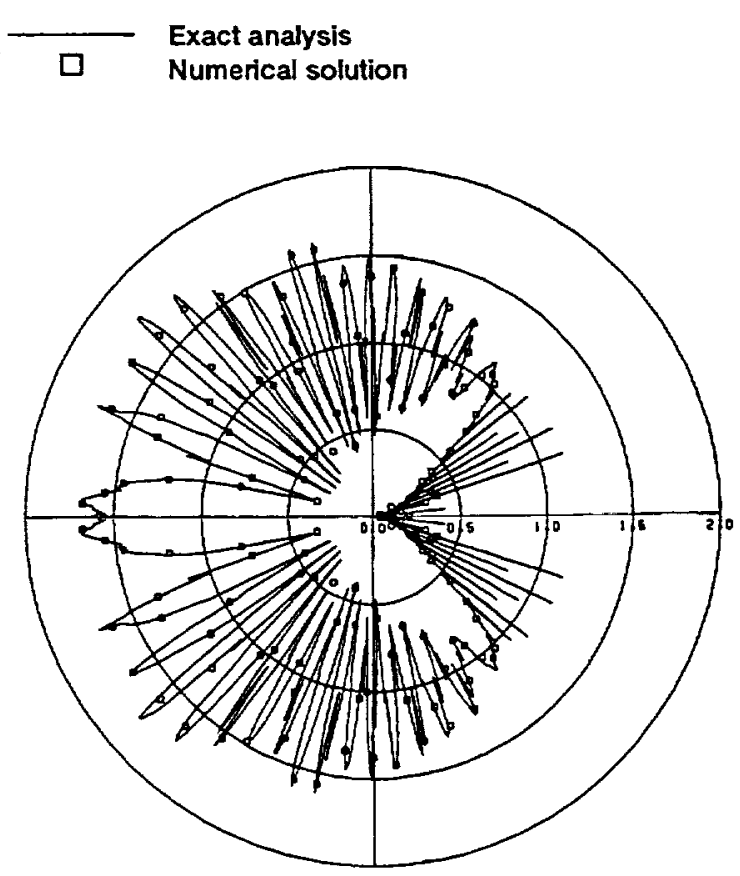

(d) Application of model ring grid to high frequency scattering $k r=150,2904$ nodes.

Figure 1.-Application of modal element methods in acoustic scattering from a hard circular cylinder. 


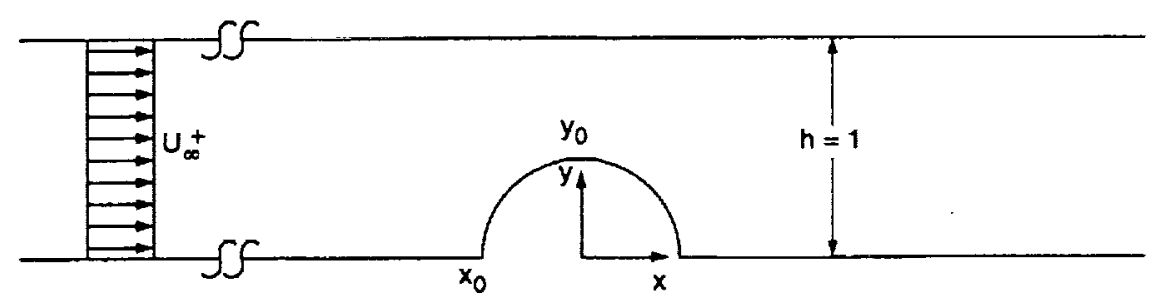

Figure 2.-Flow field geometry.

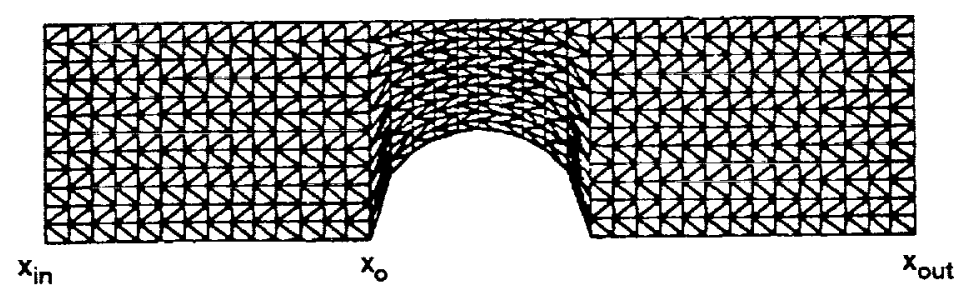

$x_{0}$

$x_{\text {out }}$

Figure 3.-Conventional finite element discretization for flow around cylindrical object in channel.

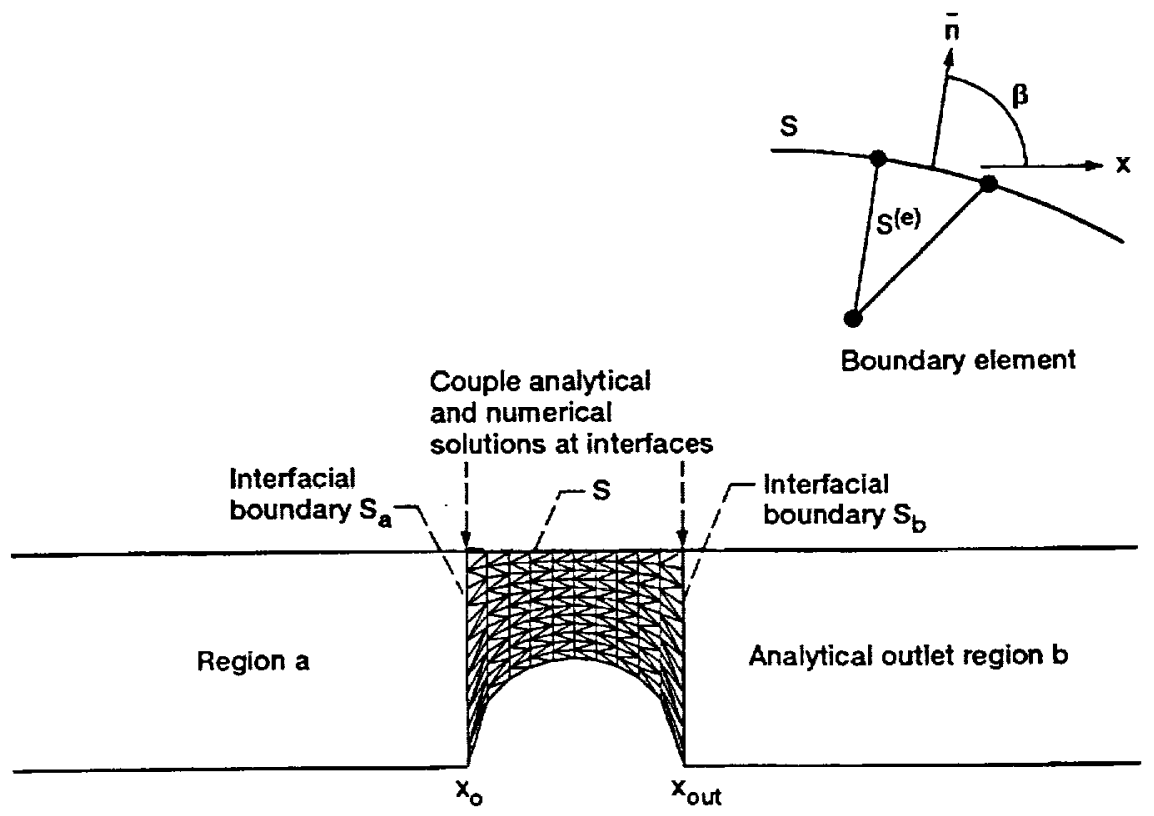

Figure 4.-Modal element finite element discretization for flow around cylindrical object in channel. 


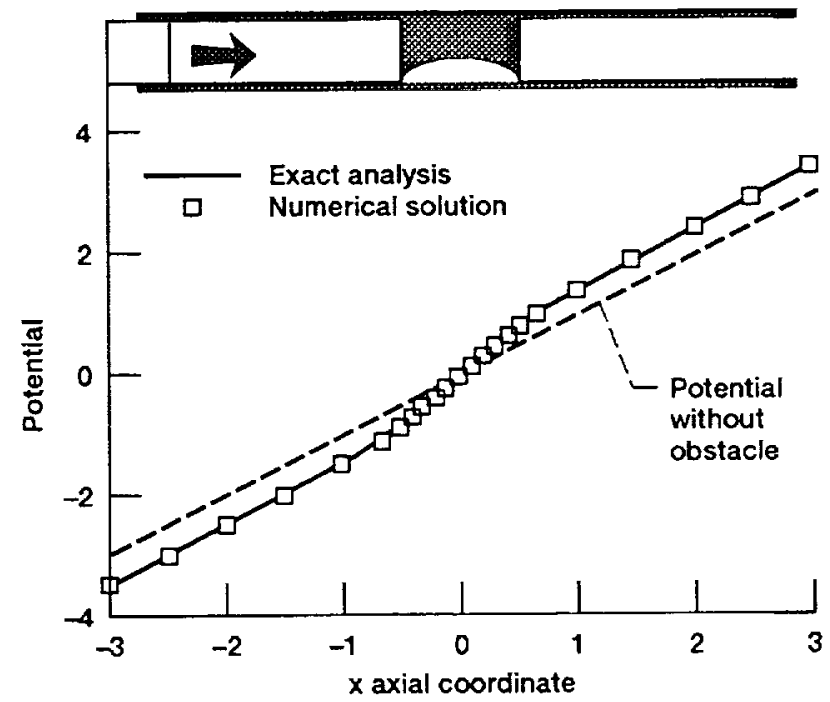

Figure 5.-Effect of a half channel obstruction on the potential along the upper wall (143 nodes and 240 elements).

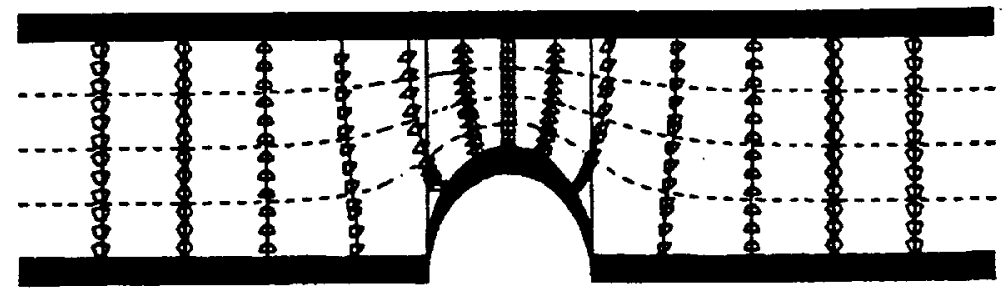

Figure 6. - Contour plots of the potential in both the finite element region and the analytical regions for the half channel obstruction (525 nodes and 960 elements).

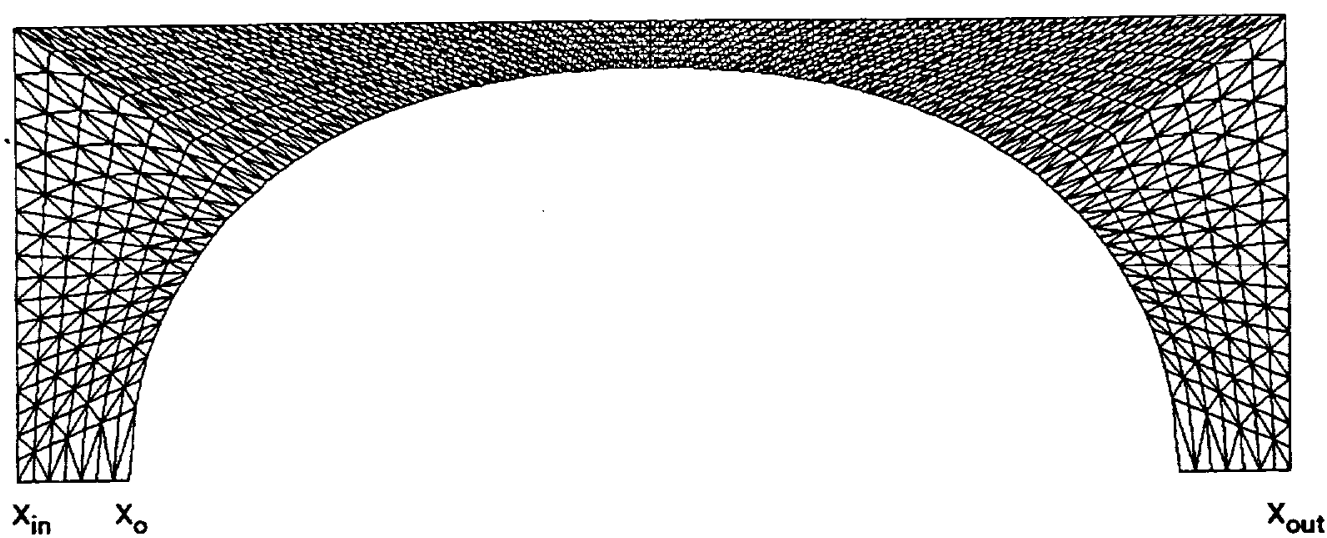

Figure 7.-Modal element finite element discretization for flow around large cylindrical object in channel. 


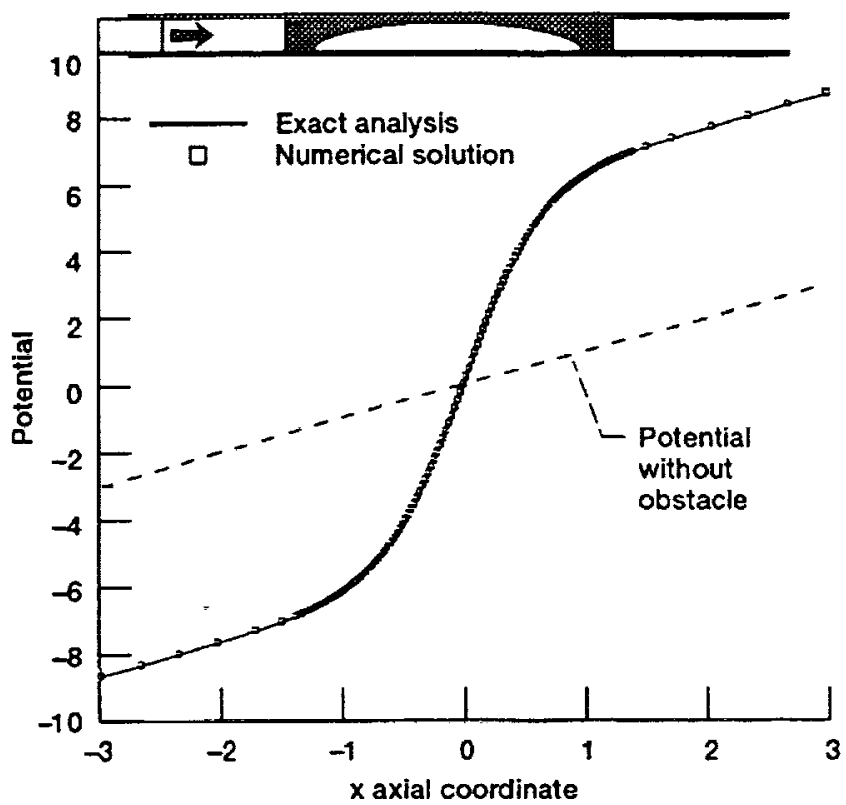

Figure 8.-Effect of a large channel obstruction on the potential along the upper wall (1272 nodes and 2212 elements).

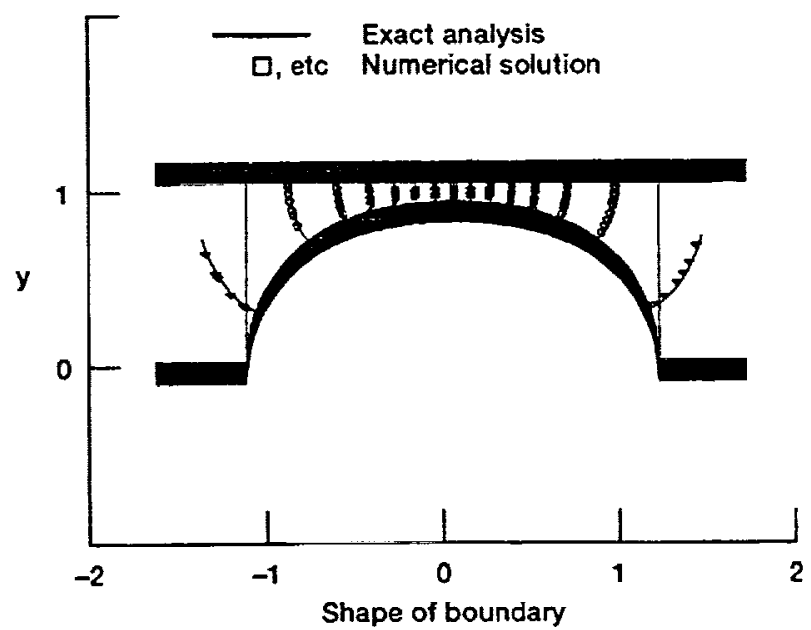

Figure 9.-Contour plots of the potential in the finite element region for the channel obstruction.

Exact analysis

$\square$, etc Numerical solution

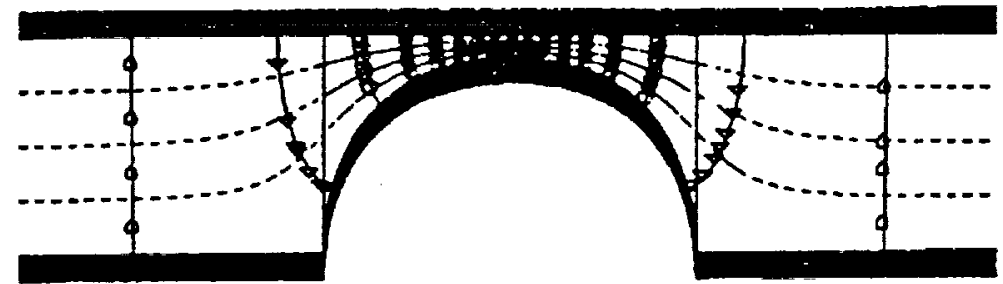

Figure 10.-Contour plots of the potential in both the finite element region and the analytical regions for the half channel obstruction (1272 nodes and 2212 elements). 
Public feporing burden for this collection of intormation is estimated to average 1 hous per response. including the time for reviewing instructions, searching existing data sources, gathering and maintaining the data needed, and cormpleting and reviewing the colection of information. Send comrrents regarding this burden estimate or any other aspect ol this Davis Highway. Suite 1204, A flington, VA 22202-4302, and to the Office of Management and Budget, Paperwork Reduction Projed (0704-0188), Washington, DC 20503.

\begin{tabular}{|l|l|l|}
\hline 1. AGENCY USE ONLY (Leave blank) & $\begin{array}{c}\text { 2. REPORT DATE } \\
\text { January } 1994\end{array}$ & $\begin{array}{r}\text { 3. REPORT TYPE AND DATES COVERED } \\
\text { Technical Memorandum }\end{array}$
\end{tabular}

\section{TITLE AND SUBTITLE}

Modal Element Method for Potential Flow in Non-Uniform Ducts:

Combining Closed Form Analysis With CFD
5. FUNDING NUMBERS

WU-505-62-52

\section{AUTHOR(S)}

Kenneth J. Baumeister and Joseph F. Baumeister

7. PERFORMING ORGANIZATION NAME(S) AND ADDRESS(ES)

National Aeronautics and Space Administration

Lewis Research Center

Cleveland, Ohio 44135-3191
8. PERFORMING ORGANIZATION REPORT NUMBER

E-8118
9. SPONSORINGMONITORING AGENCY NAME(S) AND ADDRESS(ES)

National Aeronautics and Space Administration

Washington, D.C. 20546-0001
10. SPONSORINGMONITORING AGENCY REPORT NUMBER

NASA TM-106347

AIAA-93-0813

11. SUPPLEMENTARY NOTES

Responsible person, Kenneth J. Baumeister, (216) 433-5886.

12a. DISTRIBUTIONAVAILABILTYY STATEMENT

12b. DISTRIBUTION CODE

Unclassified - Unlimited

Subject Category 64

13. ABSTRACT (Maximum 200 words)

An analytical procedure is presented, called the modal element method, that combines numerical grid based algorithms with eigenfunction expansions developed by separation of variables. A modal element method is presented for solving potential flow in a channel with two-dimensional cylindrical like obstacles. The infinite computational region is divided into three subdomains; the bounded finite element domain, which is characterized by the cylindrical obstacle and the surrounding unbounded uniform channel entrance and exit domains. The velocity potential is represented approximately in the grid based domain by a finite element solution and is represented analytically by an eigenfunction expansion in the uniform semi-infinite entrance and exit domains. The calculated flow fields are in excellent agreement with exact analytical solutions. By eliminating the grid surrounding the obstacle, the modal element method reduces the numerical grid size, employs a more precise far field boundary condition, as well as giving theoretical insight to the interaction of the obstacle with the mean flow. Although the analysis focuses on a specific geometry, the formulation is general and can be applied to a variety of problems as seen by a comparison to companion theories in aeroacoustics and electromagnetics.

\section{SUBJECT TERMS}

Finite element; Potential flow; Modes

15. NUMBER OF PAGES

\begin{tabular}{|c|c|}
\hline $\begin{array}{l}\text { 17. SECURTY CLASSIFICATION } \\
\text { OF REPORT }\end{array}$ & $\begin{array}{c}\text { 18. SECUATY CLASSIFICATION } \\
\text { OF THIS PAGE } \\
\text { Unclassified }\end{array}$ \\
Unclassified
\end{tabular}

19. SECURITY CLASSIFICATION OF ABSTRACT Unclassified
20. LIMITATION OF ABSTAACT

Standard Form 298 (Rev. 2-89)

Prescrbed by ANSI Std. Z39-18 298-102 